Barriers to recruitment included certain times of year, like public holidays. We employed the National Adult Literacy Agency to improve readability of participant documents but later, to comply with GDPR guidelines, we lengthened our Participant Information Leaflet, which some women found offputting. The trial was co-ordinated at a prestigious university and some eligible women expressed concern that they might be judged by researchers. In response, advertising through the university's website was discontinued.

Several strategies were successful. LAG members applied indepth knowledge of local communities to target recruitment to eligible women. Advertising via social media helped recruit younger women. Using a centrally-located, well-known building within each district, with good transport links, improved attendance at consent meetings, particularly important for those with mobility issues. Recruitment occurred over four waves, allowing the application of iterative learning.

Conclusion Recruitment strategies in the WCQ2 trial had variable success. Community-based trials have specific challenges such as the availability and suitability of local resources and the regulatory environment. The early and active engagement of local stakeholder groups with in-depth knowledge of communities is important, as well as the application of iterative learning.

\section{P43 DEVELOPMENT OF A CARDIOVASCULAR DISEASE RISK PREDICTION MODEL FOR POPULATION HEALTH PLANNING IN JAPAN: EPOCH-JAPAN STUDY}

${ }^{1} Y$ Murakami*, ${ }^{2} \mathrm{~T}$ Okamura, ${ }^{3,4} \mathrm{~K}$ Miura, ${ }^{3,4} \mathrm{H}$ Ueshima, on behalf of the EPOCH-JAPAN study investigators. 'Department of Medical Statistics, Toho University, Tokyo, Japan; ${ }^{2}$ Department of Preventive Medicine and Public Health, Keio Universwity, Tokyo, Japan; ${ }^{3}$ Department of Public Health, Shiga University of Medical Science, Shiga, Japan; ${ }^{4}$ Center for Epidemiologic Research in Asia, Shiga University of Medical Science, Shiga, Japan

\subsection{6/jech-2019-SSMabstracts.194}

Background Cardiovascular disease (CVD) risk prediction models are primarily used in clinical settings, but may also have potential applications in population health. For example, these models can be used to estimate 10-year CVD mortality within a region. In order to expand the applications of such models, we developed a CVD risk prediction model for population health planning that can account for temporal changes in mortality.

Methods The Evidence for Cardiovascular Prevention from Observational Cohorts in Japan (EPOCH-JAPAN) study is an individual participant data meta-analysis of cardiovascular epidemiology in the Japanese population. This project comprises 15 cohort studies involving 147,465 Japanese people, with a total of 5,543 CVD deaths. Before constructing the CVD risk prediction model for the population, we grouped these cohort studies into three categories according to their year of cohort initiation (1990-1994, 1995-1999, and 2000 or later), and used two groups for model construction (Group 1: 19901994; Group 2: 1995-1999). First, we constructed a groupspecific CVD risk prediction model based on a Cox model that included age (year), systolic blood pressure (SBP; $\mathrm{mmHg}$ ), total cholesterol (TC; $\mathrm{mg} / \mathrm{dl})$, diabetes (DM), smoking status, and study cohort as independent variables. Next, we checked the homogeneity of the model parameters using hazard ratios, and developed a common parameter using weighted mean values. Finally, to eliminate the temporal discrepancies between the model results and contemporary mortality (as of 2015), we calculated calibrating factors using government vital statistics of Japan.

Results Among the 15 cohort studies included in EPOCHJAPAN, seven were categorized into Group 1 and three were categorized into Group 2. For CVD in men, the results showed similar hazard ratios for age (1.12), SBP (1.01), and TC (1.00); furthermore, the hazard ratios were similar in both groups for DM (Group 1: 1.43, Group 2: 1.64) and current smokers (Group 1: 1.52, Group 2: 1.55). This homogeneity in model parameters was also observed in women (age: 1.14, SBP: 1.01, TC: 1.00, DM [Group 1: 1.56, Group 2: 2.27], and current smokers [Group 1: 1.63, Group 2: 1.37]), and a common parameter was developed and included in the final CVD risk prediction model. The calibrating factors to adjust for contemporary mortality in 2015 were calculated to be 0.74 in men and 0.55 in women.

Conclusion We constructed a CVD risk prediction model for population health planning that can be used to estimate current mortality in the Japanese population.

\section{P44 SEX DIFFERENCES IN THE RELATIONSHIP BETWEEN SOCIOECONOMIC STATUS AND HYPERTENSION IN FRANCE: RESULTS FROM A CROSS-SECTIONAL ANALYSIS OF THE CONSTANCES COHORT}

${ }^{1} \mathrm{~L}$ Neufcourt*, ${ }^{2} S$ Deguen, ${ }^{3,4} \mathrm{M}$ Zins, ${ }^{1} \mathrm{O}$ Grimaud. ${ }^{1}$ Depatment of epidemiology and biostatistics; 'Univ Rennes, EHESP, REPERES - EA 7449, Rennes, France; ${ }^{2}$ Department of Social Epidemiology, Institut Pierre Louis d'Epidémiologie et de Santé Publique (UMRS 1136), Paris, France; ${ }^{3}$ Paris Descartes University, Paris Descartes University, Paris, France; ${ }^{4}$ Population-Based Epidemiological Cohorts Unit, UMS 011, INSERM-UVSQ, Paris, France

\subsection{6/jech-2019-SSMabstracts. 195}

Background There is ample evidence that hypertension prevalence increases when socioeconomic status (SES) decreases. However, sex differences in this relationship has been less studied. Investigating potential sex differences could help understand the mechanisms of social health disparities. The aim of this work was to explore the pattern of associations between several indicators of SES and hypertension across sexes in a large sample of French adults.

Methods In this cross-sectional analysis, participants are adults aged between 18 and 69 years old recruited to the CONSTANCES cohort over the period 2012-2015 in 16 recruitment centers. SES was estimated using education (individual level), income (household level) and an indicator of residential socioeconomic deprivation, FDep (municipal level). Log-binomial and Poisson regressions with robust variance estimations were used to estimate the Risk Ratios (RR) comparing the extreme levels of SES and to test for interaction of sex in the associations between SES and hypertension prevalence.

Results A total of 62,247 individuals $(53 \%$ women, mean age $48 \pm 13$ years) were included. Age-standardized prevalence of hypertension was $30.1 \%$ [95\% CI $=29.7-30.6]$, higher in men $(37.3 \%[95 \% \mathrm{CI}=36.6-38.0])$ than in women $(23.2 \%[95 \%$ $\mathrm{CI}=22.7-23.8]$ ).

Globally, we found steep socioeconomic gradients of hypertension in both genders. Education showed the strongest association with hypertension prevalence, especially among women ( $\mathrm{p}$ for interaction between sex and education <0.001): ageadjusted RR comparing the lowest versus highest level of education were $1.57[95 \% \mathrm{CI}=1.47-1.68]$ in women and 1.26 $[95 \% \mathrm{CI}=1.21-1.31]$ in men. Income and FDep also displayed strong associations with hypertension, but we found no 
interaction with sex ( $\mathrm{p}$ for interaction $=0.420$ and 0.236 respectively). Age-adjusted RR comparing the lowest versus highest level of household income was 1.30 [95\%CI $=1.21-$ 1.39] and RR comparing the most versus least deprived quintile of residential areas was 1.26 [95\% CI=1.22-1.31]. Adding all the three indicators in a full model resulted in a more pronounced attenuation of the income and FDep gradients compared to education, however all the associations remain significant in men and women.

Discussion In this sample, individual education displayed different patterns of association with hypertension across sexes, but not household income nor neighborhood deprivation. This result underlines that systematic stratification by sex may not be appropriate in all analyses focusing on hypertension as outcome of interest. Besides, these results suggest that education attainment has a greater impact on women in preventing hypertension and therefore prevention should start early. More work is needed to understand sex differences in the causal pathway linking SES and hypertension.

\section{P45 HEALTHCARE SYSTEM PERFORMANCE IN CONTINUITY OF CARE FOR PATIENTS WITH SEVERE MENTAL ILLNESS: A COMPARISON OF FIVE EUROPEAN COUNTRIES}

${ }^{1} \mathrm{P}$ Nicaise*, ${ }^{2} \mathrm{D}$ Giacco, ${ }^{3} \mathrm{~A}$ Pfennig, ${ }^{4} \mathrm{~A}$ Lasalvia, ${ }^{5} \mathrm{M}$ Welbel, ${ }^{2} \mathrm{~S}$ Priebe, ${ }^{1} \mathrm{~V}$ Lorant. ${ }^{1}$ Institute of Health and Society (IRSS), Université Catholique de Louvain, Brussels, Belgium; ${ }^{2}$ Unit for Social and Community Psychiatry, Queen Mary University of London, London, UK; ${ }^{3}$ Department of Psychiatry and Psychotherapy, Technische Universität Dresden, Dresden, Germany; ${ }^{4}$ Department of Public Health and Community Medicine, University of Verona, Verona, Italy; Institute of Psychiatry and Neurology, Warsaw, Poland

\subsection{6/jech-2019-SSMabstracts. 196}

Background Patients with severe mental illness (SMI) require continuity of care. Continuity of care refers to the uninterrupted contact of patients with the service delivery system, and includes three main dimensions: cross-sectional, longitudinal, and relational continuity. Cross-sectional care continuity refers to the provision of comprehensive care during a single episode, longitudinal continuity to the provision of care over time, and relational continuity to the quality of the patientprovider relationship. In Europe, healthcare systems have developed differently from two basic models: national health (NHS) and regulated-market systems (RMS). It is unclear which healthcare system model is more performant in the delivery of continuity of care. Therefore, we examined the care provision, regulation, and financing features in two NHS - England and Veneto (Italy) - and three RMS countries Germany, Belgium, and Poland -, and assessed empirically the system's performance in cross-sectional, longitudinal, and relational care continuity.

Methods 6,418 SMI patients were recruited from psychiatric hospitals in the five countries and followed up one year after admission. Data were collected on their use of services and contact with care professionals. Care continuity was assessed using several indicators: the time gap between hospital discharge and outpatient care, access to services, number of contacts with psychiatrists and other care professions, satisfaction with care continuity, and helping alliance. Multivariate regressions were used to control for patients' characteristics and robust standard errors were computed to account for the clustering effect of the recruiting hospitals.
Results Important differences were found between healthcare systems. NHS countries, particularly Veneto, had more effective longitudinal and cross-sectional care continuity than RMS countries: patients had a longer gap between hospital discharge and outpatient care $(R R=1.71, p<0.001)$, had half the chance to access to supported living $(\mathrm{OR}=0.54, \mathrm{p}<0.05)$, and to access different professions $(\mathrm{RR}=0.76, \mathrm{p}<0.01)$. However, Germany had similar results to England, while Poland had lower performance measures, despite a mixed NHS and RMS model. Relational continuity had mixed results, patients being slightly less satisfied in RMS than in NHS countries $(\mathrm{RR}=0.90, \mathrm{p}<0.01)$ but having a higher helping alliance $(\mathrm{RR}=1.07, \mathrm{p}<0.01)$.

Conclusion Organisational features have an impact on crosssectional and longitudinal continuing care delivery. Although the relationship between healthcare provision, regulation, and financing mechanisms, and care continuity is complex to disentangle, stronger regulation of care provision and financing at a local policy level should be considered for care continuity. Yet, relational care continuity seemed less affected by organisational mechanisms.

\section{P46 TRENDS IN NATIONAL PHARMACEUTICAL EXPENDITURE ON DIABETES 2011-2015; THE RISING COST OF BLOOD GLUCOSE-LOWERING MEDICATIONS}

${ }^{1} \mathrm{KN}$ O'Neill*, ${ }^{2} \mathrm{KE}$ Bennett, ${ }^{1} \mathrm{SM}$ McHugh, ${ }^{1} \mathrm{AP}$ Fitzgerald, ${ }^{1} \mathrm{PM}$ Kearney. ${ }^{1}$ School of Public Health, UCC, Cork; ${ }^{2}$ RCSI, Dublin

\subsection{6/jech-2019-SSMabstracts. 197}

Background Medication costs are the primary driver of increasing medical expenditure on diabetes. We explore trends in pharmaceutical expenditure on diabetes between 2011 and 2015, examining the impact of newer blood glucose-lowering medications, and estimating the effect of cost-containment measures implemented during this time.

Methods Data from a national pharmacy claims database were analysed. Patients dispensed items used in the treatment or management of diabetes were identified. Total expenditure associated with diabetes was calculated by extracting data on all diabetes-related items dispensed to eligible patients. Costs were categorised into two groups; diabetes-specific items include items used directly in the treatment of diabetes and other items include all other condition-related. The impact of two specific cost-containment measures, co-payments and reference pricing, implemented over the study period were assessed using segmented linear regression analyses of interrupted time series.

Results Total expenditure varied over the study period, peaking at $€ 216,994,441$ in 2012. Expenditure on diabetes-specific items increased steadily by $18 \%$ reaching $€ 153,621,477$ in 2015, with blood glucose-lowering medications accounting for $73 \%$ of this increase. During the same period, expenditure on other items decreased by $32 \%$ to $€ 50,835,856$. The introduction of reference pricing for atorvastatin in November 2013 resulted in immediate costs savings of $€ 2.4$ million per yearly quarter (level change $\mathrm{p}<0.001$ ).

Conclusion The increasing costs of blood glucose-lowering medications overshadows the effect of cost-containment measures. Innovative policies are required to ensure high quality diabetes care can be provided at an equitable, affordable and sustainable rate. 